\title{
A Nonlinear Strategy to Control Unstable Underactuated Mechanical Systems with Underactuation > 1. Applications to Control Augmentations ${ }^{\dagger}$
}

\author{
J.Á. Acosta* and M. López-Martínez
}

Dept. de Ingeniería de Sistemas y Automática, Escuela Técnica Superior de Ingenieros, Universidad de Sevilla, Camino de los Descubrimientos s/n, 41092, Sevilla, Spain

\begin{abstract}
The essence of the complex dynamics originated by the interaction between the pilot- $\&$-aircraft can be captured on labs, by means of unstable-underactuated mechanical systems. Thus, the unactuated links describe the autonomous aircraft dynamics (flight control system) and the actuated the manual one (pilot). It is well-known that, in some flight conditions, the pilot-\&-aircraft interaction can render the system unstable. Thus, a standard solution of designing a decoupled dynamics between the flight control system and the pilot, either becomes useless or restrict the maneuverability unnecessarily. The nonlinear and compact strategy proposed here can pave the way to a less conservative (or even safety) solution in aircraft implementation. Successful experiments on the Furuta's pendulum are reported and to the best of authors' knowledge it has the largest attraction basin experimentally tested so far.
\end{abstract}

\section{INTRODUCTION}

10 years ago, K.J. Åström proposed that the essence of the complex control problem originated by the joint of the pilot-\&-aircraft can be captured on labs, by means of unstable underactuated mechanical systems $[1,2]$. Thus, the unactuated links describe the autonomous aircraft dynamics (flight control system) and the actuated the manual one (pilot). In this way, the proposed approach deals with the control of piloted vehicles whose autonomous behaviour is open-loop unstable and can be modelled by means of unstable underactuated mechanical systems [1-3].

In applications where an operator is present, such as cars, ships and aircrafts, the operator's mission is to stabilise the system in a small neighborhood of the desired position, where the pilot imposes the reference velocities. In particular, in aeronautics, stability and control augmentation systems are introduced to improve handling (or flying) qualities on the aircraft allowing precise control with low pilot workload. In this way, control augmentation systems are sometimes additionally required to provide a particular type of response to pilot's set points. The flight control system has a critical mission because in some flight conditions (including landing and take off) the unstable mode is so fast that a pilot cannot stabilise the system. Moreover, for the automatic control the presence of a pilot is a complication because the pilot may also drive the system unstable through manual control actions. An excellent example of the latter was the unstable JAS 39 Gripen aircraft which was proved to be unstable in some flight conditions under the small rate limitation nonlinearity of the pilot

*Address correspondence to these authors at the Dept. de Ingeniería de Sistemas y Automática, Escuela Técnica Superior de Ingenieros, Universidad de Sevilla, Camino de los Descubrimientos s/n, 41092, Sevilla, Spain; E-mails: jaar@esi.us.es and mlm@esi.us.es

${ }^{\dagger}$ This work was supported by The Consejería de Innovacíon Ciencia y Empresa of The Junta de Andalucía under IAC programme. actuation [4]. A typical and motivational example is the control of a VTOL aircraft $[5,6]$. So, the flight control system should thus fulfill the dual task of stabilizing the aircraft without restricting the maneuverability unnecessarily. The design of control strategies for such situations is a significant challenge as was pointed out in [7].

In fact, the complex dynamics induced by the joint pilot$\&$-aircraft makes the manufacturers encounter severe difficulties to the control problem. The usual solution adopted is to divide the available control authority between control and stabilization by means of hybrid control and decoupling the dynamics, but as pointed above this design can either be useless or restrict the maneuverability unnecessarily. In this approach a nonlinear controller is proposed whose nonlinear behavior decides the control authority autonomously. The nonlinear control strategy is designed by using the classical geometric framework of feedback linearization [8] which allow us to construct an output that renders the system to be a minimum-phase system, even taking into account friction and drag forces. Moreover, the solution is explicitly given in a compact form for the whole class. The proposed solution improves the solution given in $[1,2]$ since the automatic control is independent of the saturation limit of the manual control, i.e. the stability of the autonomous part of the system is not altered by manual control, due to the minimum-phase property. This solution reveals a great benefit for understanding and testing the control of open-loop unstable systems and, in particular it can pave the way to a less conservative (or even safety) solution in future aircraft implementation.

\section{Outline}

In [9] we showed an explicit solution of a class of underactuation mechanical systems of underactuation degree one. After that, in [10] we generalize that result for the underactuation degree larger than one. Thus, in the next 
section, we briefly mention all necessary previous results of [10], without proofs. Nevertheless, since most of results referenced here are an extension to the case of underactuated mechanical systems of underactuation degree one, we refer to readers to [9]. Then, a coupled of sections to extend the class solved in [10] by means of the singularly perturbed theory, removing and relaxing some of the assumptions. Finally an applications section with examples and successful experimental results and a conclusion section.

\section{Notation}

Throughout the paper all vectors are column vectors, even the gradient operator $\nabla_{x}=\frac{\partial}{\partial x}$, and the hessian $\nabla_{x}^{2}=\frac{\partial^{2}}{\partial x^{2}}$. For vector functions $F: \mathbb{R}^{n} \rightarrow \mathbb{R}^{m}$, we define the matrix $\nabla_{x} F(x)=$ $\left[\nabla_{x} F_{1}(x), \ldots, \nabla_{x} F_{m}(x)\right]$. When clear from the context the subindex of the operator $\nabla$ and the arguments of the functions will be omitted. For matrices $M \succ 0, M \in \mathbb{R}^{m \times n}$, means symmetric and positive definite. Acronyms used: r.h.s. means right hand side; i.e. means that is.

\section{PRELIMINARY RESULTS}

Let $(q, \dot{q}) \in \mathbb{R}^{n} \times \mathbb{R}^{n}$ be the generalized coordinates and velocities, respectively, of an underactuated mechanical systems, i.e. there are fewer control inputs than degrees of freedom. The Lagrange's equations read

$M(q) \ddot{q}+C(q, \dot{q}) \dot{q}+D(\dot{q})+\nabla U(q)=\left[\begin{array}{ll}O & I_{m}\end{array}\right]^{\top} \tau$

where $M \in \mathbb{R}^{n \times n}$ is the symmetric and positive definite inertia matrix, $U \in \mathbb{R}$ is the potential function, $\tau \in \mathbb{R}^{m}$ the number of independent control inputs, the matrix $C \in \mathbb{R}^{n \times n}$ contains the Coriolis and centrifugal forces, and it can be calculated as

$C(q, \dot{q}) \dot{q}=\left[\nabla_{q}(M \dot{q})-\frac{1}{2} \nabla_{q}(M \dot{q})^{\top}\right] \dot{q}$,

the matrix $D(\dot{q}) \in \mathbb{R}^{n}$ is the vector of friction and/or drag forces. In fact, the class considered refers to systems with underactuation degree $(n-m)$, with $m \geq 1$. Then, we partition intuitively the set of generalized coordinates $q=$ $\left(q_{1}, q_{2}\right) \in \mathbb{R}^{n-m} \times \mathbb{R}^{m}$, where the unactuated degree of freedom is represented by the $q_{1}$-coordinate and the actuated ones by the set of the generalized $q_{2}$-coordinates. After this partition the Lagrange's equations of motion can be written as

$$
\left[\begin{array}{cc}
M_{11} & M_{12}^{\top} \\
M_{12} & M_{22}
\end{array}\right] \ddot{q}+\left[\begin{array}{c}
F_{1}(q, \dot{q}) \\
F_{2}(q, \dot{q})
\end{array}\right]=\left[\begin{array}{c}
0 \\
I_{m}
\end{array}\right] \tau,
$$

where now $M_{11} \in \mathbb{R}^{(n-m) \times(n-m)}, M_{12} \in \mathbb{R}^{m \times(n-m)}, M_{22} \in \mathbb{R}^{m \times m}$ and we have introduced the scalar and vector functions $F_{1}(q, \dot{q})$ $\in \mathbb{R}^{n-m}$ and $F_{2}(q, \dot{q}) \in \mathbb{R}^{m}$, respectively. The first step of the approach presented here is to linearize partially the equations of motion (1), as done in [11] where it was called collocated partial feedback. Indeed, after some simple calculations the partially feedback-linearized system takes the affine in control form:

$$
\begin{aligned}
& F(q, \dot{q}) \triangleq\left[\begin{array}{c}
-M_{11}^{-1} F_{1}(q, \dot{q}) \\
O
\end{array}\right], \quad G(q) \triangleq\left[\begin{array}{c}
-M_{11}^{-1} M_{12}^{\top} \\
I_{m}
\end{array}\right] \\
& \ddot{q}=F(q, \dot{q})+G(q) u,
\end{aligned}
$$

and the vector function $F_{1}(q, \dot{q})$ was defined as

$F_{1}(q, \dot{q}) \triangleq\left(I_{n-m} O\right)[C(q, \dot{q}) \dot{q}+D(\dot{q})+\nabla U(q)]$,

where as usual in mechanical systems the matrix $M_{11}$ is uniformly positive definite, and then invertible and $\operatorname{rank}\left(M_{12}\right)=n-m($ see $[11])$.

The assumptions for the class solved in [10] were:

A.1. (Definition of the class)

-The argument of the elements of the inertia matrix are $M_{11}\left(q_{1}\right), M_{12}\left(q_{1}\right)$ and $M_{22}\left(q_{2}\right)$ or constant matrix.

The potential function is of the form

$$
U(q) \triangleq V\left(q_{1}\right)+v\left(q_{2}\right)
$$

-The nonlinear friction and/or drag forces $D_{1}(\dot{q}) \dot{q} \geq$ 0 with $D_{1}(\dot{q}) \triangleq\left(I_{n-m} O\right) D(\dot{q})$, satisfies a linear growth bound only on the unactuated coordinates

$$
\left\|D_{1}(\dot{q})\right\| \leq \gamma\left\|\dot{q}_{1}\right\|, \forall \dot{q}_{1} \in \mathcal{D} \subseteq \mathbb{R}^{n-m}
$$

where, $\forall 0$ is a positive constant.

A.2. (Rank condition) $m \geq(n-m)$.

A.3. (Underactuated coordinates) The unstable equilibria of the unactuated coordinates $\left(q_{1_{*}}\right)$ are isolated in $\Omega$, i.e. $\nabla^{2} V\left(q_{1 *}\right)<0$ and $\nabla V\left(q_{1}\right) \neq 0, \forall q_{1} \in \Omega \backslash\left\{q_{1 *}\right\}$.

A.4. (Integrability condition) Let $\nabla m_{i}=\left(\nabla m_{i}\right)^{\top}$ with $m_{\mathrm{i}}\left(q_{1}\right)$, $i=1 \ldots m$, are the rows of the matrix $M_{12}$.

Let us denote throughout the paper to help the reader to identify quickly the zero dynamics, the state space of these zero dynamics as $Z=\left(q_{1}, \dot{q}_{1}\right)^{\top}$ and its equilibrium as $Z_{*}$. Thus, the state space will be, from now on, $(Z, \eta)^{\top}$ and, with $\eta$ denoting the output of the system. In [10] and under the above assumptions we proved among other results that the composite smooth-static-feedback controller ${ }^{1}$ given by

$u=\Delta^{-1}\left(K_{1}\left(-M_{12} M_{11}^{-1} F_{1}+\dot{M}_{12} \dot{q}_{1}\right)+K_{2} M_{12} \dot{q}_{1}-v\right)$

$v=-K_{3}\left(M_{12} \dot{q}_{1}+K_{4} \tilde{\eta}\right)$,

with $\Delta \triangleq K_{1} M_{12} M_{11}^{-1} M_{12}^{\top}-I_{m}$, and the constant and full rank $(m \times m)$-matrices satisfying the conditions

C1. $K_{1} \succ 0$, and such that the matrix $\bar{M}\left(q_{1}\right) \succ 0$ for all $q_{1}$ $\in \Omega \subseteq \mathbb{R}^{n-m}$, where $\bar{M} \triangleq M_{12}^{\top} K_{1} M_{12}-M_{11}$;

C2. $K_{2} \succ 0$ and $k_{2}>\gamma$, with $k_{2}$ the minimal eigenvalue of the full-rank matrix $M_{12}^{\top} K_{2} M_{12}, \forall q_{1} \in \Omega$;

C3. $K_{3} \succ 0$, and diagonal; and

C4. $K_{4} \succ 0$,

${ }^{1}$ The derivation of the controller is identical to the $m-n=1$ given in [9]. It is omitted here for brevity. 
ensure that, for all $(Z, \eta) \in \Omega \times \mathcal{D} \times \mathbb{R}^{m}$, the closed-loop system is partially state feedback input-output linearizable through the output ${ }^{2}$

$$
\tilde{\eta} \triangleq\left[K_{1} M_{12} I_{m}\right] \dot{q}+K_{2} \int_{q_{1 *}}^{q_{1}} M_{12}(\mu) \cdot d \mu-\eta_{*}
$$

The Assumptions A.1 and A.2 played a critical role in the stabilization problem since they allowed us to find out an energy-like Lyapunov function for the zero dynamics. On the other hand, these conditions does not play a key role for the (local) stabilization and therefore we use this fact here to extend the class. We will show that the proposed controller renders a (local) asymptotical and exponential stability of the desired equilibrium of the extended class, i.e. the singularlyperturbed one.

\section{The Control Problem}

Recalling that the main control objective is to maneuver manually the set point (emulating aircraft flying), i.e. a manual operation, then $\eta$ denotes the actuated part (piloted). In this way the control problem to be addressed is first to stabilise the equilibrium $\left(Z_{*}, \eta_{*}\right)^{\top}$ and then a trajectory tracking.

\section{THE SINGULARLY PERTURBED CLASS}

In this section we prove that through the output (10) the equilibrium of the extended class, called singularlyperturbed class, can also be stabilised, and moreover, the assumptions A.1 and A.2 can be relaxed as follows:

A.1' on one hand, in A.1 the matrix $\mathrm{M}_{22}$ can be function of the unactuated coordinates; and

A.2' on the other hand, the A.2 is completely removed.

Only an additional assumption is needed, to generalize this result:

Assumption A.5 $\left[\frac{\partial M_{22}\left(q_{1}\right)}{\partial q_{1}}\right]_{q_{1}=q_{1 *}}=0$.

The above Assumption is necessary to assure that the desired equilibrium of the zero dynamics $Z_{*}$ is not modified at least locally, for an arbitrary set point $\eta_{*}$. It is also noticeable that in the particular case of regulation at $\eta_{*}=O$ this assumption is not needed at all, but we state the general case.

Proposition 1. Consider the underactuated mechanical system (4) under the Assumptions A.1-A.5 with the relaxations made in A.1' and A.2', and with the matrices $K_{i}$, $i=1, \ldots, 4$ satisfying the conditions $\mathrm{C} 1-\mathrm{C} 4$. Then, the following assertions hold:

the closed-loop system is partially state feedback input-output linearizable in the set $(Z, \eta) \in \Omega \times \mathcal{D}$ $\times \mathbb{R}^{m}$ through the output (10) through the smoothstatic feedback given by (8).

\footnotetext{
${ }^{2}$ Notice that, since $q_{1}$ is a vector we have introduced some abuse of notation, only for compactness. See the TCP example further to clarify the computation in the case of a MIMO system.
}

(ii) the external controller (9) assures that there exits a real number $k_{3}^{*}>0$ such that, for $k_{3}>k_{3}^{*}$ the systems is singularly perturbed and so, all trajectories starting in a compact ball remain bounded, where $k_{3}$ stands for the maximal eigenvalue of the matrix $K_{3}$. Moreover, the desired equilibrium $\left(Z_{*}, \eta_{*}\right)$ is (locally) asymptotically (exponentially) stable.

Proof. The first claim follows directly following the usual procedure of calculating the derivative of (10), and becomes

$$
\begin{aligned}
\tilde{\eta} & =\left[K_{1} M_{12} I_{m}\right] \ddot{q}+K_{1} M_{12} \dot{q}_{1}+K_{2} M_{12} \dot{q}_{1} \\
& =-K_{1} M_{12} M_{11}^{-1} M_{12}^{\top} F_{1}-u+K_{1} M_{12} \dot{q}_{1}+K_{2} M_{12} \dot{q}_{1}=v,
\end{aligned}
$$

and isolating the controller $u$ we get (8).

To prove the second claim, we first prove that the zero dynamics associated to the proposed output is locally exponentially stable. These zero dynamics can be obtained after expanding the first $(n-m)$ equations of (4) using the controller derived in (8) with $v=0$. Thus, tedious but straightforward calculations show that under the assumptions made the linearized zero dynamics round the equilibrium becomes $\bar{M}_{*} \delta \ddot{q}_{1}\left(M_{12}^{\top} K_{2} M_{12}-\nabla D_{1}+\Gamma\right)_{*} \delta \dot{q}_{1}-\nabla^{2} V_{*} \delta q_{1}=O$, where we defined the matrix $\Gamma \triangleq \nabla\left(M_{12}^{\top} \dot{q}_{2}\right)-\nabla\left(M_{12}^{\top} \dot{q}_{2}\right)^{\top}$ and the variables for the linearization of the zero dynamics as $\delta q_{1} \triangleq q_{1}-q_{1 *}$, and we used the notation $(\cdot)_{*}$ for the matrices evaluated at the equilibrium $Z_{*}$. By $\mathrm{C} 2$ we fix $M_{12}^{\top} K_{2} M_{12}>\gamma$ and by A.1 $\left\|\nabla D_{1}\right\|<\gamma$ then the damping matrix, i.e. $M_{12}^{\top} K_{2} M_{12}-\nabla D_{1} \succ 0$. Additionally, $\bar{M}_{*} \succ 0$ and by A.3 $\left(-\nabla^{2} V\right)_{*} \succ 0$. Thus, since $\Gamma$ is skew-symmetric and all the remaining matrices are positive definite then, from the results of linear theory [12] we know that for these second order and linear dynamics the equilibrium $z_{*}$ of the zero dynamics is locally asymptotically and exponentially stable. ${ }^{3}$

Now, we prove the last part regarding to the singular perturbations theory, using the external controller given by

(9) and just invoking the result given in [13, Th. 11.4]. ${ }^{4}$ For, we rewrite the closed-loop dynamics qualitatively in the following way

$$
\begin{aligned}
& \dot{z}=\psi(Z, \tilde{\eta}) \\
& \varepsilon \dot{\tilde{\eta}}=\left(O M_{12}\right) Z-K_{4} \tilde{\eta},
\end{aligned}
$$

where $\psi(\cdot)$ is a smooth function, the small parameter $\varepsilon \triangleq$ $1 / k_{3}$. When $\varepsilon=0$ the roots of (12) are $\tilde{\eta}=h(Z)=$ $-K_{4}^{-1} M_{12} \dot{q}_{1}$ with $h(O)=O$. Thus, the boundary-layer system can be obtained defining the variable $y \triangleq \tilde{\eta}-h(Z)$, and then the equations (11)-(12) in the new coordinates become

$$
\begin{aligned}
& \frac{d Z}{d t}=\psi(Z, y-h(Z)) \\
& \frac{d y}{d \tau}=-K_{4} y+\mathcal{O}(\varepsilon)
\end{aligned}
$$

\footnotetext{
${ }^{3}$ Locally Exponentially Stable (LES).

${ }^{4}$ This result is an extension to infinite interval of time of the well-known Tikhonov's Theorem.
} 
with $\tau=\left(t-t_{0}\right) / \varepsilon$ the new time scale. First notice that, on the one hand the reduced system $(\varepsilon=0)$, in this case, is exactly the zero dynamics which we actually know that its equilibrium is LES, and, on the other hand the so-called boundary-layer system is described by (14) and its equilibrium is also LES uniformly in $Z$. Therefore all the conditions from [13, Th. 11.4] are satisfied, and then there exists an $\varepsilon^{*}>0$ such that for all $\varepsilon<\varepsilon^{*}$ the equilibrium of the entire system is LES on the compact ball where all the conditions are satisfied.

Remark 2. Notice that, in the proposition 1, we could not invoke the well-known results from [8] because we cannot use the crucial fact that the dynamics on the $\tilde{\eta}$-coordinate is linear, and so neither in the $v$-coordinate.

\section{ASYMPTOTIC OUTPUT TRACKING}

The propositions 1 assure that, at least, the equilibrium of the whole system is LES. This result is very promising because that means certain robustness to small disturbances. In this way, with a slight modification of the external controller (9) we can also assure a bounded asymptotic output tracking to a prescribed reference function $\eta_{*}(t)$. We state this result with only a sketch of the proof because is based on the one given in [8, Prop. 4.5.1].

Proposition 3. Consider the underactuated mechanical system (4) under the Assumptions A.1-A.5 with the relaxations made in A.1' and A.2', and with the matrices $K_{i}$, $i=1, \ldots, 4$ satisfying the conditions C1-C4. Suppose further that $\eta_{*}(t)$ and $\dot{\eta}_{*}(t)$ are defined for all $t \geq 0$ and bounded. Then, the smooth-static-feedback controller (8) and the external controller

$$
v=r . h . s .(9)-K_{3} K_{4} K_{3} \int_{q_{1 *}}^{q_{1}} M_{12}(\mu) \cdot d \mu+\dot{\eta}_{*}(t)
$$

assure a bounded asymptotic output tracking [8].
Proof. [Sketch] We define a new output $\chi \triangleq \tilde{\eta}+$ $K_{3} \int_{q_{1 *}}^{q_{1}} M_{12}(\mu) d \mu$, which is a (local) diffeomorphism to $\tilde{\eta}$, and then the external $\tilde{\eta}$-dynamics becomes $\dot{\chi}=-K_{3} K_{4} \chi$, remaining the zero dynamics unchanged. Since the closedloop system is in the form given in [8, Prop. 4.5.1] and all the conditions stated there are satisfied then same arguments hold here.

Remark 4. We underscore here that, the controller for the zero dynamics (8) is independent of the rate saturation of the manual control (pilot). Thus, the controllers (9) and (15) could have been designed with a saturated $v$, as $v=\operatorname{sat}($ r.h.s. of (9)) or $v=\operatorname{sat}($ r.h.s. of (15)), with $\operatorname{sat}(\cdot)$, any standard saturation function, without modifying the stability of the zero dynamics. Clearly, the solution proposed here improve those from $[1,2]$.

\section{EXAMPLES AND EXPERIMENTS}

We test the approach via simulations in one example and via experiments in the available laboratory equipment. Since, the applicability of the approach include also multi input/output systems we include a two-coupled pendula as an example, while the laboratory equipment that has been used for the experiments is Furuta's pendulum. It should be notice that the two-coupled pendula is of underactuation degree two in contrast with the last application we propose, the rotary pendulum or so-called the Furuta pendulum, which is of underactuation degree one. In addition, when designing controllers for model-based underactuated systems usually the proposed controllers are based on models neglecting the friction forces, even in the unactuated coordinates. However, in [14] the importance of the friction to stabilise this system was shown. So, in the approach proposed here, the friction forces can be taken into account

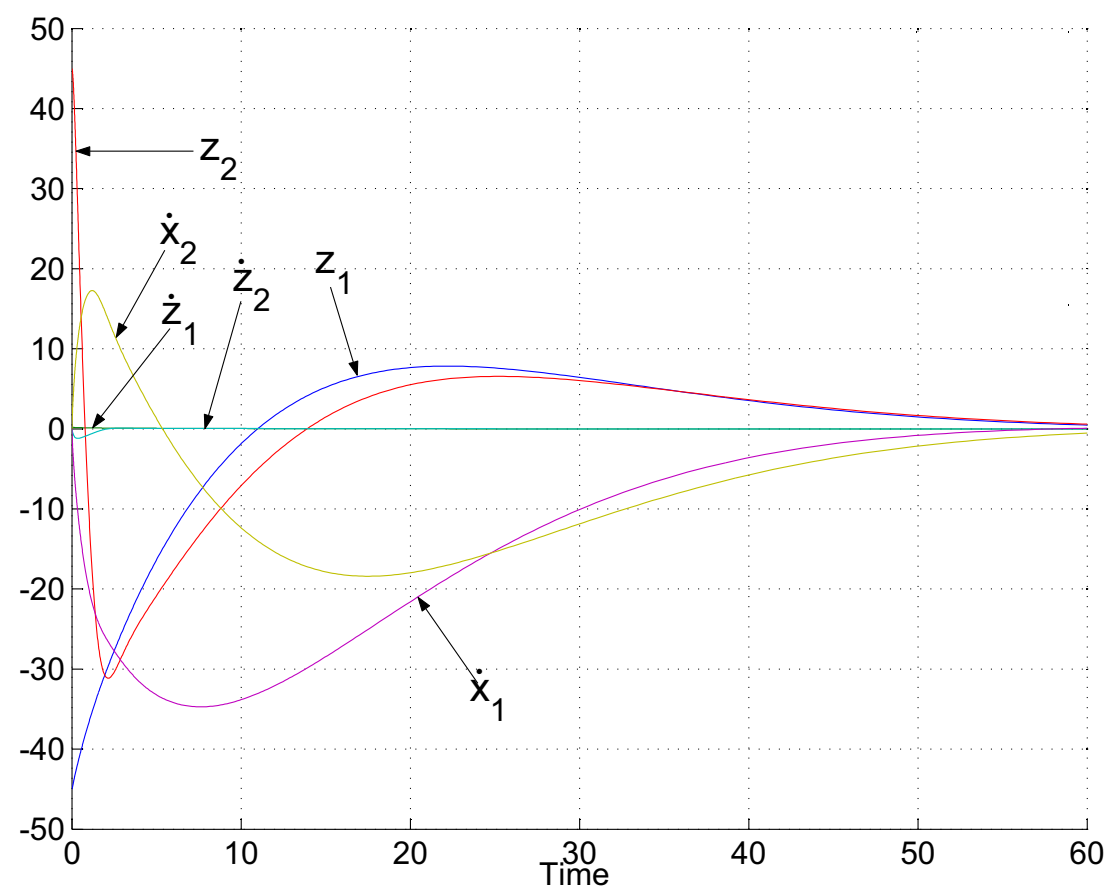

Fig. (1). Simulations of two-coupled pendula. 
Table 1. Physical Parameters of the Systems

\begin{tabular}{|c|c|c|c|c|c|c|}
\hline \multirow{2}{*}{$\begin{array}{c}\text { Dynamical } \\
\text { System }\end{array}$} & \multirow{2}{*}{$\frac{\text { DOF }}{n}$} & \multicolumn{2}{|c|}{ Potential $U(q)$} & \multicolumn{3}{|c|}{ Inertia Matrix $M(q)$} \\
\hline & & $V\left(q_{1}\right)$ & $1\left(q_{2}\right)$ & $M_{11}\left(q_{1}\right)$ & $\boldsymbol{M}_{12}^{\top}\left(\boldsymbol{q}_{1}\right)$ & $M_{22}(q)$ \\
\hline TCP & 4 & $m g\left(l_{1} c z_{1}+l_{2} c z_{2}\right)$ & - & $m\left[\begin{array}{ll}l_{1}^{2} & 0 \\
0 & l_{1}^{2}\end{array}\right.$ & $m\left[\begin{array}{ll}l_{1} c z_{1} a & l_{1} c z_{1} b \\
l_{2} c z_{2} b & l_{2} c z_{2} a\end{array}\right.$ & $(M+2 m) I_{2}$ \\
\hline FP & 2 & $m g l c z$ & - & $J_{p}$ & mrlcz & $J_{a}+m r^{2}+J p s^{2} z$ \\
\hline
\end{tabular}

easily in the dynamical model. Notice that we only are interested in stabilising the upper position of the pendulum, not to swing-up from any position, whose solution was reported in $[15,16]$. To the best of the authors knowledge the solution proposed by singular perturbations enlarges the largest region of attraction obtained so far, even with friction. In theory, this solution could stabilise the upper position from any point over the upper half plane. The excellent performance and the large region of attraction has been tested in the actual laboratory pendulum and reported here. Table 1 presents a summary of the relevant physical parameters of the systems related to the potencial function and the inertia matrix. The outputs $\eta$, internal control laws $u$ and external controllers $v$ are obtained by substituting in the equations (10), (8) and (9), respectively.

It is interesting to underscore the easy tuning of the controller (8)-(9) due, mainly, to its physical meaning. In fact, on one hand from (8) we see that the control gains $K_{1,2}$ are related with the internal dynamics (autonomous control) and, on the other hand from (9) the control gains $K_{3,4}$ are related with the external dynamics (manual control, pilot). Finally, to ease the reading, let us present the examples in the more intuitive physical coordinates $(Z, \dot{x})$, since the change of coordinates given by $(Z, \eta) \leftrightarrow(Z, \dot{x})$ is a (global) diffeomorphism.

\section{The Two-Coupled Pendula (TCP)}

This system consists of a platform than can be moved on the plane $x-y$, and two pendula which are mounted on the platform. The pendula are positioned with an angle shift of $90^{\circ}$ respect to $z$-axis and orientated respect to the $x$-axis with an angle of $30^{\circ}$. In Table 1 , the following parameters have been defined $a=\cos 30^{\circ}, b=\sin 30^{\circ}$, the masses of the pendula are $\mathrm{m}=1 \mathrm{~kg}$, the lengths are $l_{1}=10 \mathrm{~m}, l_{2}=1 \mathrm{~m}$, the viscous friction constants of the unactuated degrees of freedom are $F=1 \mathrm{~N} \cdot \mathrm{m} /(\mathrm{rad} / \mathrm{s})$ and $g=10 \mathrm{~m} / \mathrm{s}^{2}$. The system has 4 degrees of freedom, the $\mathrm{x}$ and $\mathrm{y}$ coordinates and the angles of each pendulum respect to the $z$-axis, $\varphi_{1}$ and $\varphi_{2}$. This platform can be actuated with two forces orientated in $\mathrm{x}$-axis and $\mathrm{y}$-axis respectively. In this way, the system has two unactuated degrees of freedom $\varphi_{1}$ and $\varphi_{2}$, which will be denoted as $z_{1}$ and $z_{2}$ respectively. In Fig. (1) the results of a simulation are shown. Notice the excellent overshoot and also that the controller avoid the collision between both pendula. The control gains have been selected as follows: $K_{1}$ $=30 I_{2}, K_{2}=100 I_{2}, K_{3}=I_{2}$ and $K_{4}=I_{2}$. A couple of videos of this simulation are available at http://www.esi2.us.es/ jaar/investiga_e.htm. The redesign of the output has been calculated by introducing the following line integral in (10) as

$$
\int_{\left(z_{1}, z_{2}\right) .}^{\left(z_{1}, z_{2}\right)} M_{12} d \mu=\left[a \sin \left(z_{1}\right)+b \sin \left(z_{2}\right), b \sin \left(z_{1}\right)+a \sin \left(z_{2}\right)\right]
$$

\section{Experimental Results on Furuta's Pendulum (FP)}

The model of the rotary pendulum, or so-called Furuta's pendulum, used in this article is thoroughly described in [15] and [16]. The values of the physical parameters-summarized in Table $\mathbf{1}$ - used in the experimental framework are: mass of the pendulum $=0.0679(\mathrm{Kg})$, length of the pendulum $=$ $0.28(\mathrm{~m})$, radius of the arm $=0.235(\mathrm{~m})$, mass of the arm $=$ $0.2869(\mathrm{Kg})$, constant torque $=7.4$, moment of inertia of the motor $=0.0012\left(\mathrm{Kg} \cdot \mathrm{m}^{2}\right)$. The full control system is shown in Fig. (2). The laboratory electro-mechanical system is composed by: a DC motor (15 Nm / 2000 rpm) with tachometer that measures the speed of the arm; a power supply (50 VA); a PWM servo-amplifier; a pendulum; an encoder that measures the angle of the pendulum and a slip ring that drives the signal to the base. The control system is composed by: a monitor PC with a target (DS1102) for control based on DSP (TMS320C31) and a software (DSPACE) for control, monitoring and supervisor. In this practical

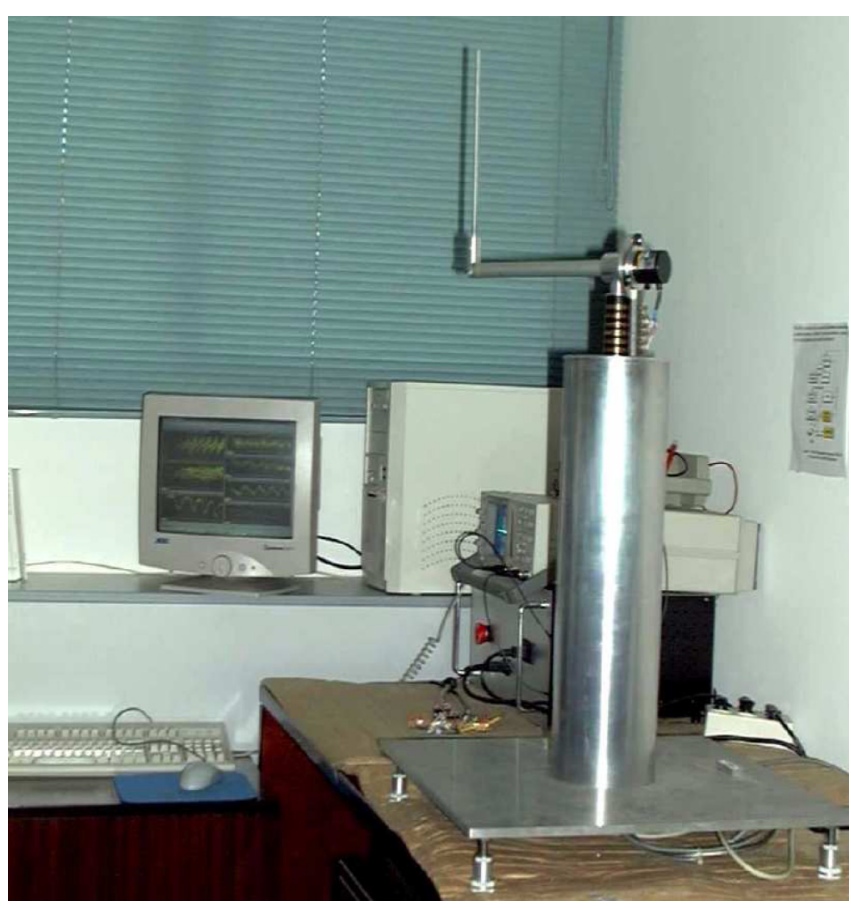

Fig. (2). Laboratory pendulum. 


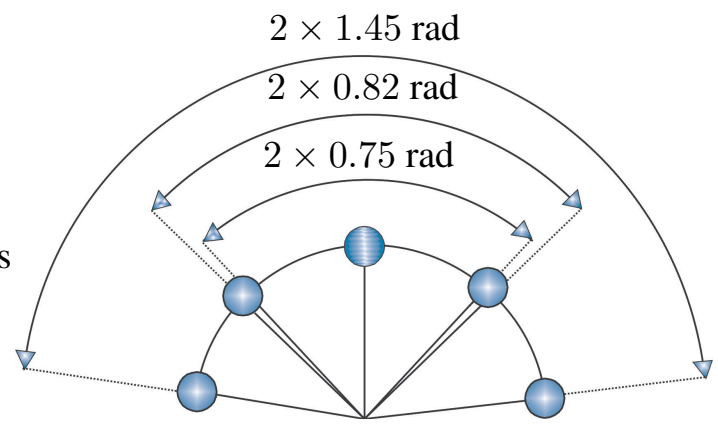

Fig. (3). Experimental basin of attraction.

case, since in this approach the friction in the actuated coordinates can be compensated then, we include a nonlinear compensator based on the LuGre model [17] to dominate the friction forces of the arm of the pendulum, in the linearizing controller $u$. The controller gains for the experiments were $K_{1}=100, K_{2}=500, K_{3}=10(\varepsilon=0.1$, from proposition 1) and $K_{4}=25$. In Fig. (4) the initial conditions for the angular position of the pendulum was $z(0)$ $=1.45 \mathrm{rad}$. To the best of our knowledge this is the largest region of attraction achieved in experimental results to stabilise this kind of pendulum. An approximation to the maximum theoretical value of $z(0)$ can be obtained through the condition $\operatorname{det}(\Delta(z))=0$, i.e. $K_{1}\left(m r l / J_{p}\right) \cos ^{2} z=1$, whose value for the $K_{1}$ given is $1.5 \mathrm{rad}$. In fact, we also would like to compare with the maximum theoretical values obtained by passivity methods. In [18] the maximum theoretical $z$ is given by the equation $|z|<\arcsin \left(\sqrt{r^{2} /\left(r^{2}+l^{2}\right)}\right.$. In [19] the condition which gives the largest admissible angle $|z| \leq$ $\operatorname{arcos}\left(1+\left(m r l / J_{p}\right)^{2}\right)^{-\frac{1}{2}}$. In both cases the formulas are not tunable, since depends only on the physical parameters, and gives rise in our pendulum to a maximum $z(0)=0.7 \mathrm{rad}$ and $z(0)=0.9 \mathrm{rad}$ respectively, which are approximately the half of the value presented above. In Fig. (3) we show an schematic of the experimental results obtained with the approach given $z(0)=1.45 \mathrm{rad}$; with the passive approach $z(0)=0.82 \mathrm{rad}$; and with an Linear Quadratic Regulator $(\mathrm{LQR})$ where $z(0)=0.75 \mathrm{rad}$. The similar results obtained with the LQR and with the passive method are due to the friction forces which make the close-loop system non passive as commented before (see [14, 20]). Fig. (4) shows an experiment where the initial conditions for velocities are not near to zero. From the theoretical point of view, the region of attraction could tend to the horizontal position of the pendulum, by increasing $K_{1}$. Unfortunately, the system saturates and it was not possible to enlarge more this practical region of attraction. The saturation of the input
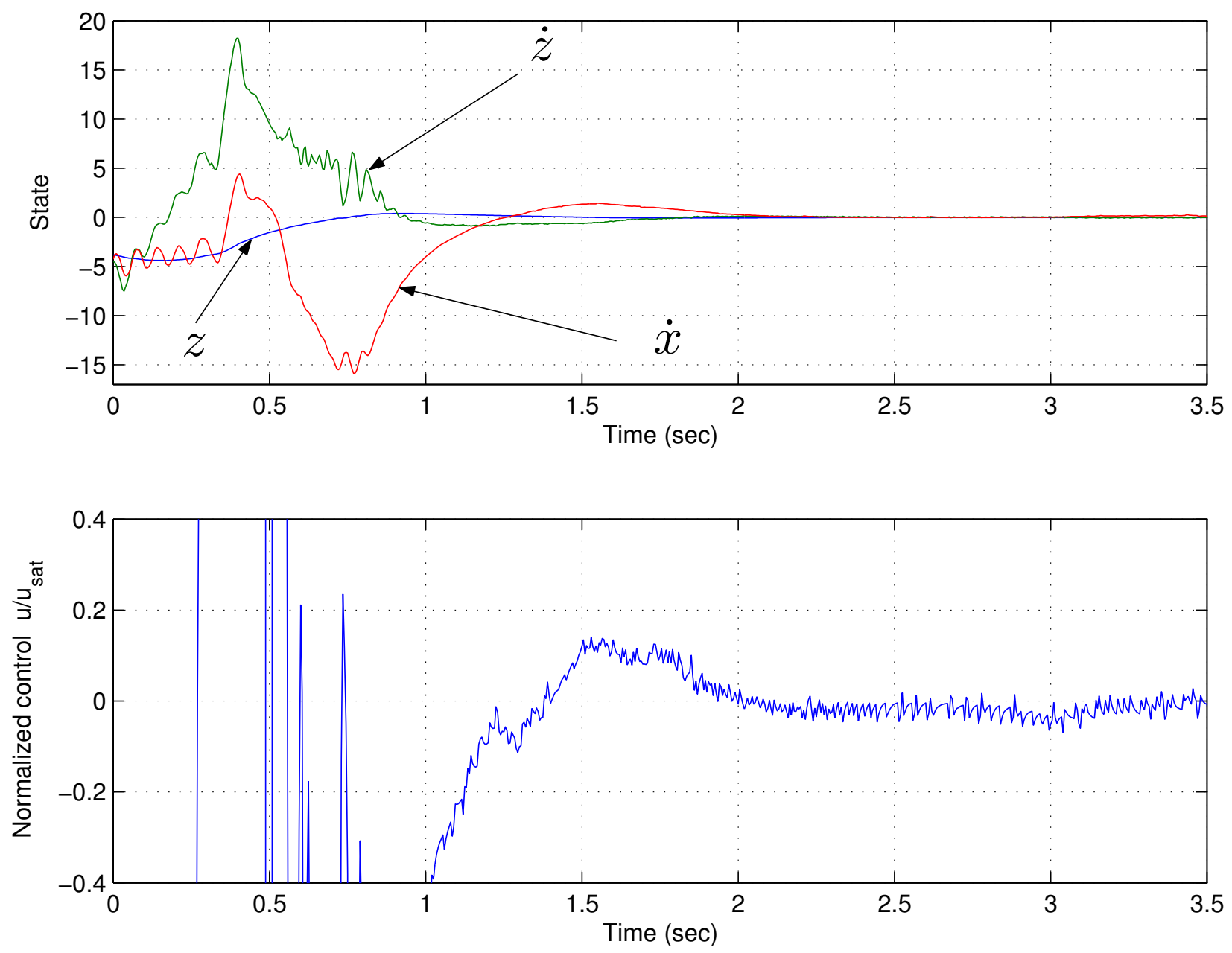

Fig. (4). Furuta pendulum: regulation experiment. 

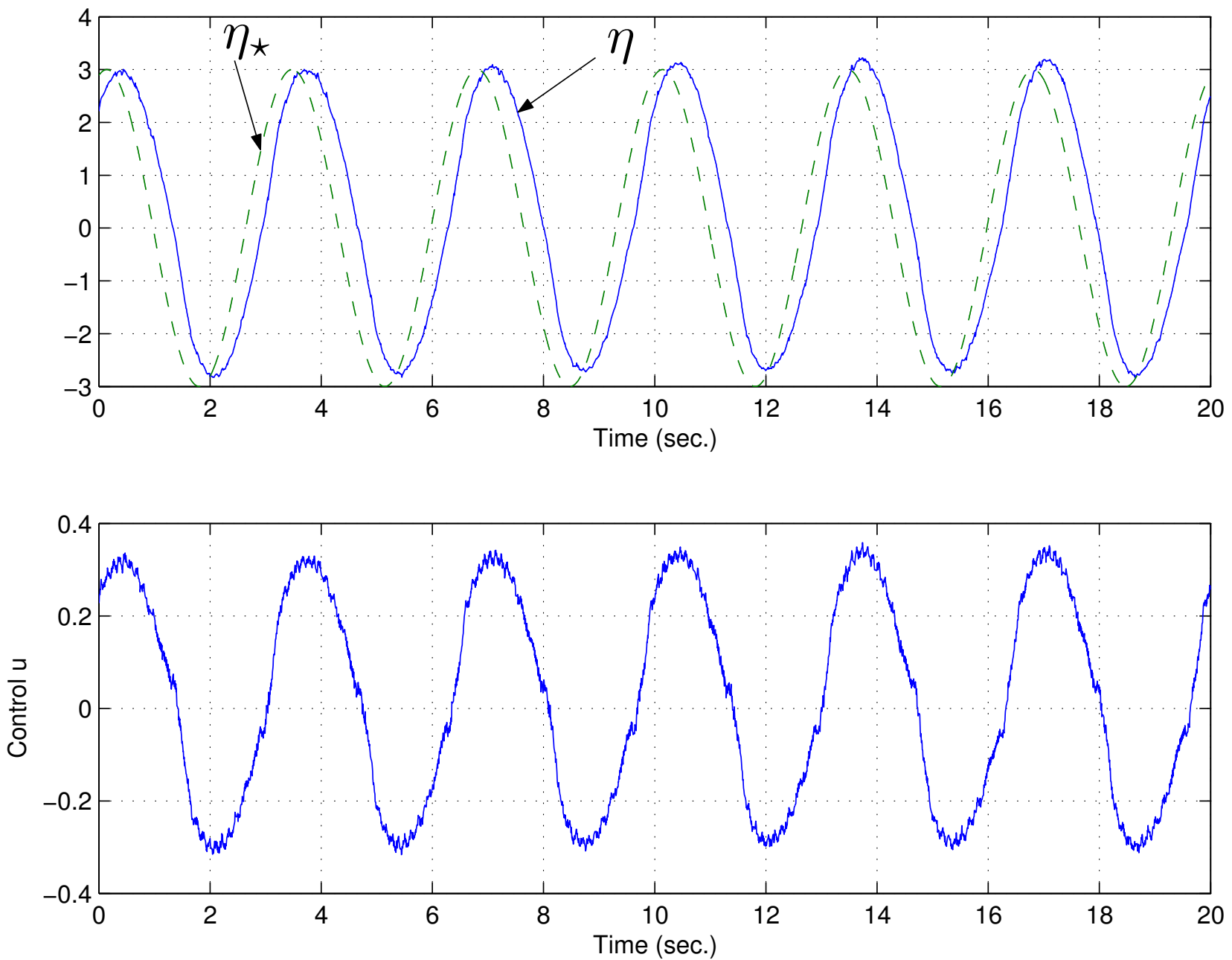

Fig. (5). Furuta pendulum: tracking experiment.

control is shown for both experiments in the figures. Notice that even with saturation the objective was achieved.

We also report and experiment tracking a sinusoidal reference in Fig. (5) with the controller given in the proposition 3. In this experiment a joystick was used emulating the manual control of a pilot. This experiment shows the robustness of the approach. Notice that the references were given in the actuated link (the arm), and the controller was able to track a sinusoidal reference in the arm and at the same time keep the pendulum at upright position. This situation emulates, from a control point of view, the same situation of a flight control system which has to follow the references of the pilot and, at the same time, takes care about the stability of the aircraft.

\section{CONCLUSIONS}

The complex dynamics induced by the joint of the pilot$\&$-aircraft can be described by means of unstable underactuated mechanical systems, where the unactuated part describes the autonomous aircraft dynamics and the actuated one the piloted. This paper presents an output to control unstable underactuated mechanical systems with underactuation degree even larger than one, which yields an explicit controller, for the whole class, that renders the closed-loop system to be minimum-phase. The approach is supported by singular perturbations theory. Successful experimental results on Furuta's pendulum are reported which, to the best of authors' knowledge, presents the largest attraction basin experimentally tested so far, and has been compared experimentally with another linear and non linear controllers. The approach can yield a great benefit for understanding and testing strategies to control the unstable aircraft, and it can pave the way to a less conservative (or even safety) solution in aircraft implementation and, it can provide to us new directions on how to control the interaction dynamics without the conservative solutions.

\section{ACKNOWLEDGEMENTS}

The authors would like to thank to Full Professors E. Ponce and E. Freire both at Dept. of Applied Mathematics, and to Full Professor M. Rodríguez-Danta at the Dept. of Applied Physics, all of them from the University of Seville, Spain, for insightful and fruitful discussions.

\section{REFERENCES}

[1] J. Åkesson and K.J. Åström, "Safe manual control on the Furuta pendulum". Control Applications, Proceedings of the IEEE Conference pp. 890-895, 2001.

[2] K.J. Åström and S.M. Brufani, "Control of an Unstable System with a Saturating Actuator". Decision and Control, Proceedings of the 36th IEEE Conference, pp. 964-965, 1997. 
[3] A. Isidori, Robust autonomous guidance: an internal model approach. Springer, London, 2003.

[4] L. Rundqwist and R. Hillgren, Phase Compensation of Rate Limiters in JAS 39 Gripen. AIAA Paper, pp. 96-3368, 1996.

[5] D. Bates and I. Postlethwaite, Robust Multivariable Control of Aerospace Systems, DUP Science, 2002.

[6] S. Sastry, Nonlinear Systems: Analysis, Stability and Control, Springer-Verlag, New York, 1999.

[7] G. Stein, "Respect the unstable -bode lecture". In Proceedings of the 28th Conference on Decision and Control, 1989.

[8] A. Isidori, Nonlinear Control Systems, Springer, 1995.

[9] J.Á. Acosta and M. López-Martínez, "Constructive feedback linearization of mechanical systems with friction and underactuation degree one". In Proceedings of the 45th IEEE European Control Conference, vol. 1, pp. 4255-4260, 2007.

[10] J.Á. Acosta and M. López-Martínez, "Input-output linearization on underactuated mechanical systems". 2008, (Submitted to journal).

[11] M.W. Spong, "Underactuated mechanical systems". in Control Problems in Robotics and Automation, B. Siciliano and K. Valavanis, Eds. Springer-Verlag, vol. 230, 1998. LNICS.

[12] M. Roseau, Vibrations in Mechanical Systems: Analytical Methods and Applications. Springer-Verlag, 1987.

[13] H. K. Khalil, Nonlinear Systems, Prentice Hall, Upper Saddle River, NJ, 2002.

[14] F. Gómez-Estern, A.J. van der Schaft and J.Á. Acosta, "Passivation of underactuated systems with physical damping”. In Proceedings 6th IFAC Symposium on Nonlinear Control Systems, pp. 1-3, 2004.

[15] J.Á. Acosta, J. Aracil and F. Gordillo, "On swinging the Furuta pendulum up based on Routh's reduction and SG method". In Proceedings of the 9th Mediterranean Conference on Control and Automation, Dubrovnik, Croatia, vol. 1, 2001.

[16] F. Gordillo, J.Á. Acosta and J. Aracil, "A new swing- up law for the Furuta pendulum". International Journal of Control, vol. 76, no. 8, pp. 836-844, 2003.

[17] C. Canudas de Wit, H. Olsson, K.J. Åström and P. Lischinsky, "A new model for control of systems with friction". IEEE Transactions on Automatic Control, vol. 40, pp. 419-425, 1995.

[18] A.M. Bloch, N.E. Leonard and J.E. Marsden, "Stabilization of the Pendulum on a Rotor Arm by the Method of Controlled Lagrangians". Robotics and Automation, 1999. Proceedings of the 39th IEEE Conference, vol. 1, pp. 500-505, 1999.

[19] G. Viola, R. Ortega, R. Banavar, J.Á. Acosta and A. Astolfi, "Total energy shaping control of mechanical systems: Simplifying the matching equations via coordinate changes", Automatic Control, IEEE Transactions, vol. 52, no. 6, pp. 1093-1099, 2007.

[20] C. Woolsey, C.K. Reddy, A.M. Bloch, D.E. Chang, N.E. Leonard and J.E. Marsden, "Controlled Lagrangian systems with gyroscopic forcing and dissipation", European Journal of Control, vol. 10(5), pp. 478-496, 2004.

(C) Acosta and López-Martínez; Licensee Bentham Open.

This is an open access article licensed under the terms of the Creative Commons Attribution Non-Commercial License (http://creativecommons.org/licenses/by$\mathrm{nc} / 3.0 /$ ), which permits unrestricted, non-commercial use, distribution and reproduction in any medium, provided the work is properly cited. 